\title{
On the convergence and character spectra of compact spaces
} by

\author{
István Juhász (Budapest) and William A. R. Weiss (Toronto)
}

\begin{abstract}
An infinite set $A$ in a space $X$ converges to a point $p$ (denoted by $A \rightarrow p$ ) if for every neighbourhood $U$ of $p$ we have $|A \backslash U|<|A|$. We call $c S(p, X)=\{|A|: A \subset X$ and $A \rightarrow p\}$ the convergence spectrum of $p$ in $X$ and $c S(X)=\bigcup\{c S(x, X): x \in X\}$ the convergence spectrum of $X$. The character spectrum of a point $p \in X$ is $\chi S(p, X)=$ $\{\chi(p, Y): p$ is non-isolated in $Y \subset X\}$, and $\chi S(X)=\bigcup\{\chi S(x, X): x \in X\}$ is the character spectrum of $X$. If $\kappa \in \chi S(p, X)$ for a compactum $X$ then $\{\kappa, \operatorname{cf}(\kappa)\} \subset c S(p, X)$.

A selection of our results ( $X$ is always a compactum):

(1) If $\chi(p, X)>\lambda=\lambda^{<\widehat{t}(X)}$ then $\lambda \in \chi S(p, X)$; in particular, if $X$ is countably tight then $\chi(p, X)>\lambda=\lambda^{\omega}$ implies that $\lambda \in \chi S(p, X)$.

(2) If $\chi(X)>2^{\omega}$ then $\omega_{1} \in \chi S(X)$ or $\left\{2^{\omega},\left(2^{\omega}\right)^{+}\right\} \subset \chi S(X)$.

(3) If $\chi(X)>\omega$ then $\chi S(X) \cap\left[\omega_{1}, 2^{\omega}\right] \neq \emptyset$.

(4) If $\chi(X)>2^{\kappa}$ then $\kappa^{+} \in c S(X)$, in fact there is a converging discrete set of size $\kappa^{+}$in $X$.

(5) If we add $\lambda$ Cohen reals to a model of GCH then in the extension for every $\kappa \leq \lambda$ there is $X$ with $\chi S(X)=\{\omega, \kappa\}$. In particular, it is consistent to have $X$ with $\chi S(X)=\left\{\omega, \aleph_{\omega}\right\}$.

(6) If all members of $\chi S(X)$ are limit cardinals then $|X| \leq\left(\sup \left\{|\bar{S}|: S \in[X]^{\omega}\right\}\right)^{\omega}$.

(7) It is consistent that $2^{\omega}$ is as big as you wish and there are arbitrarily large $X$ with $\chi S(X) \cap\left(\omega, 2^{\omega}\right)=\emptyset$.

It remains an open question if, for all $X, \min c S(X) \leq \omega_{1}$ (or even $\left.\min \chi S(X) \leq \omega_{1}\right)$ is provable in $\mathrm{ZFC}$.
\end{abstract}

1. Introduction. Let us start by recalling that a (transfinite) sequence $\left\langle x_{\alpha}: \alpha<\kappa\right\rangle$ is said to converge to a point $x$ in the topological space $X$ if for every neighbourhood $U$ of $x$ there is an index $\beta<\kappa$ such that $x_{\alpha} \in U$ whenever $\beta \leq \alpha<\kappa$. As usual, this is denoted by $x_{\alpha} \rightarrow x$. In this paper we shall only consider the convergence of one-to-one sequences of cardinal length.

It is also customary to define when an infinite subset $A$ of $X$ converges to the point $x$ (in symbols: $A \rightarrow x$ ). This means that for every neighbourhood

2010 Mathematics Subject Classification: 54A20, 54A25, 54A35, 54D30.

Key words and phrases: compact space, first countable space, convergent sequences and sets, character of a point. 
$U$ of $x$ we have $|A \backslash U|<|A|$. Note that if $X$ is a compactum, i.e. an infinite compact Hausdorff space, then $A \rightarrow x$ is equivalent to $x$ being the unique complete accumulation point of $A$.

Obviously, if the one-to-one sequence $\left\langle x_{\alpha}: \alpha<\kappa\right\rangle$ converges to $x$ then so does its range $\left\{x_{\alpha}: \alpha<\kappa\right\}$ as a set. Conversely, if $|A|=\kappa$ is a regular cardinal and $A \rightarrow x$ then every sequence of order type $\kappa$ that enumerates $A$ in a one-to-one manner converges to $x$ as well.

However, for singular $\kappa$ this converse is simply false. Indeed, let $\kappa$ be singular with $\lambda=\operatorname{cf}(\kappa)<\kappa$ and consider the following topology on $\kappa+1$ : All points $\alpha<\kappa$ are isolated and the neighbourhoods of the last point $\kappa$ are of the form $(\kappa+1) \backslash A$ where $A \subset \kappa$ and $|A| \leq \lambda$. Clearly, in the resulting space $X$ every subset of $\kappa$ of size $\kappa$ converges to the last point $\kappa$ but no one-to-one sequence of order type $\kappa$ does. More generally, if $Y$ is any Hausdorff extension of $X$ and $y$ is any point of $Y$ different from the point $\kappa$ then for some $Y$-neighbourhood $U$ of $y$ we have $|U \cap \kappa| \leq \lambda$, hence such a sequence cannot converge to $y$ as well. Note that we could take as $Y$ any Hausdorff compactification of $X$, showing that, even in compacta, we can have a converging set of singular size such that no one-to-one sequence enumerating it in the order type of its cardinality converges.

We are now ready to give the definition of the convergence spectrum both locally, at a point, and globally, in the whole space. So fix a topological space $X$ and a point $p \in X$.

DeFINITION 1.1.

$$
c S(p, X)=\{|A|: A \subset X \text { and } A \rightarrow p\}
$$

is the convergence spectrum of $p$ in $X$. Moreover,

$$
c S(X)=\bigcup\{c S(x, X): x \in X\}
$$

is the convergence spectrum of $X$.

It is well-known and easy to see that if $\chi(p, X)=\psi(p, X)=\kappa$ for a nonisolated point $p$ in the space $X$ then there is a $\kappa$-type one-to-one sequence in $X$ converging to $p$, hence both $\chi(p, X) \in c S(p, X)$ and $\operatorname{cf}(\chi(p, X)) \in$ $c S(p, X)$. It is also well-known that $\chi(p, X)=\psi(p, X)$ for every point $p$ of a compactum $X$. Consequently, $\{\chi(p, X), \operatorname{cf}(\chi(p, X))\} \subset c S(p, X)$ whenever $p$ is non-isolated in the compactum $X$. In particular, the convergence spectrum of a compactum always contains a regular cardinal.

Note that if $X$ is regular and $p \in Y \subset X$ then $\chi(p, Y)=\chi(p, \bar{Y})$. Consequently, if $X$ is a compactum and $p$ is non-isolated in the subspace $Y \subset X$ then $\chi(p, Y) \in c S(p, \bar{Y}) \subset c S(p, X)$ as well. This motivated us to introduce the following definition.

Definition 1.2. For a non-isolated point $p$ of the space $X$ we let

$$
\chi S(p, X)=\{\chi(p, Y): p \text { is non-isolated in } Y \subset X\}
$$


and we call $\chi S(p, X)$ the character spectrum of $p$ in $X$. Moreover,

$$
\chi S(X)=\bigcup\{\chi S(x, X): x \in X \text { non-isolated }\}
$$

is the character spectrum of $X$.

It follows from our considerations above that $\chi S(p, X) \subset c S(p, X)$ whenever $p$ is a non-isolated point in a compactum $X$. It is also obvious that we have $\omega \in \chi S(p, X)$ iff $\omega \in c S(p, X)$ but we shall see that this equivalence fails for uncountable cardinals.

There have been several questions around that may be conveniently formulated using the convergence or character spectra of compacta. Perhaps the oldest such problem, raised by M. Hušek, can be put as follows: Is $\min c S(X) \leq \omega_{1}$ for every compactum $X$ ? Of course, this is equivalent to asking if in every compactum there is either a convergent $\omega$-sequence or a convergent $\omega_{1}$-sequence.

Let us note here that, as is shown by any non-isolated point in a countable subset, we trivially have

$$
\min c S(X) \leq \min \chi S(X) \leq 2^{\omega}
$$

for every compactum $X$. Consequently, the answer to Hušek's question is trivially YES under $\mathrm{CH}$.

A. Dow proved in [6] that if one adds any number of Cohen reals to a model of $\mathrm{CH}$ then in the resulting extension, while the continuum is as large as you wish, Hušek's question is still answered affirmatively.

Actually, it is still open if, for any compactum $X$, even the stronger inequality $\min \chi S(X) \leq \omega_{1}$ is provable in ZFC. This would easily follow from the following conjecture that was formulated in [13]: In every countably tight compactum there is a point of character $\leq \omega_{1}$. It was shown in [13] that if one adds $\omega_{1}$ Cohen reals to any ground model then this conjecture holds in the resulting generic extension.

The following very interesting and natural question was raised by Arhangel'skii and Buzyakova in [2] (we reformulate it using our terminology): Assume that for a compactum $X$ we have $\varrho \notin c S(X)$ for every uncountable regular cardinal $\varrho$. (Such spaces were called $K$-compacta in [9].) Is then $X$ first countable?

We note that $X$ is first countable iff $\chi S(X)=\{\omega\}$. Clearly, for a $\mathrm{K}$ compactum $X$ we have $\operatorname{cf}(\lambda)=\omega$ for every $\lambda \in \chi S(X)$. A space with this apparently weaker property was called an $A B$-compactum in [9], and the problem if an AB-compactum is a K-compactum was also raised in [9]. One of our main results, Corollary 3.7 below, gives consistent counterexamples to this problem.

The following local version of the above Arhangel'skii-Buzyakova question, in a different disguise, was first raised in [3] and then, apparently inde- 
pendently, in [2]: If for a point $p$ in the compactum $X$ we have $\varrho \notin c S(p, X)$ for every uncountable regular cardinal $\varrho$, is then $\chi(p, X)$ countable? Kunen gave a negative answer to this question by constructing, in ZFC, compacta with such points of uncountable character. In fact, the methods of [19] and 20. can be used to construct a compactum with such a point of character $\lambda$ for every singular cardinal $\lambda$ of countable cofinality.

In sharp contrast to this, an affirmative answer can consistently be given to the above global question of Arhangel'skii and Buzyakova. In fact, in [9] it was shown under the assumption $2^{\omega}<\aleph_{\omega}$ that even all AB-compacta are first countable (see Corollary 2.9 below). On the other hand, the consistent examples of non-K AB-compacta, provided by Corollary 3.7, clearly cannot be first countable.

2. Inclusion in spectra. We start by recalling that a transfinite sequence $\left\langle x_{\alpha}: \alpha<\kappa\right\rangle$ is said to be a free sequence in the space $X$ if for every $\alpha<\kappa$ we have

$$
\overline{\left\{x_{\beta}: \beta<\alpha\right\}} \cap \overline{\left\{x_{\beta}: \alpha \leq \beta<\kappa\right\}}=\emptyset .
$$

Clearly, any free sequence is one-to-one and it is also easy to see that, as a subset, every free sequence is discrete. In $T_{1}$ spaces every countable discrete set is free.

For any space $X$, we denote by $\widehat{F}(X)$ the smallest cardinal $\kappa$ for which there is no free sequence of size $\kappa$ in $X$. By a well-known result of Arhangel'skii, a compactum $X$ is countably tight iff there are no uncountable free sequences in $X$, that is, iff $\widehat{F}(X)=\omega_{1}$. On the other hand, it was shown in [17] that if in a compactum $X$ there is a free sequence of length $\varrho=\operatorname{cf}(\varrho)>\omega$ then there is also one that converges. So the following simple result implies that if $X$ is a compactum with $\widehat{F}(X)>\varrho=\operatorname{cf}(\varrho)>\omega$ then $\varrho \in \chi S(X)$; in particular, if $X$ is not countably tight then $\omega_{1} \in \chi S(X)$.

Proposition 2.1. If the free sequence $\left\langle x_{\alpha}: \alpha<\kappa\right\rangle$ in the $T_{2}$ space $X$ converges to the point $p$ then

$$
\psi\left(p, \overline{\left\{x_{\alpha}: \alpha<\kappa\right\}}\right)=\operatorname{cf}(\kappa) .
$$

Proof. Clearly, $x_{\alpha} \rightarrow p$ implies that even $\psi\left(p,\left\{x_{\alpha}: \alpha<\kappa\right\} \cup\{p\}\right) \geq$ $\operatorname{cf}(\kappa)$. On the other hand, since $\left\langle x_{\alpha}: \alpha<\kappa\right\rangle$ is free we have

$$
\overline{\left\{x_{\alpha}: \alpha<\kappa\right\}} \backslash\{p\}=\bigcup_{\alpha<\kappa} \overline{\left\{x_{\beta}: \beta<\alpha\right\}},
$$

and this equality obviously implies $\psi\left(p, \overline{\left\{x_{\alpha}: \alpha<\kappa\right\}}\right) \leq \operatorname{cf}(\kappa)$.

Free sequences and the cardinal function $\widehat{F}$ play an important role in the following technical but very basic result of ours as well. 
Lemma 2.2. Assume that $X$ is a $T_{3}$ space and $\varrho, \mu$ are cardinals such that $\widehat{F}(X) \leq \varrho \leq \operatorname{cf}(\mu)$, and moreover $p \in X$ with $\psi(p, X) \geq \mu$. Then either

(i) there is a discrete set $D \in[X]^{<\varrho}$ with $p \in \bar{D}$ and $\psi(p, \bar{D}) \geq \mu$, or

(ii) there is a discrete set $D \in[X]^{\varrho}$ such that $D \rightarrow p$.

Proof. Assume that (i) fails, so that for every discrete $D \subset X$ with $|D|<\varrho$ we have $p \notin \bar{D}$ or $\psi(p, \bar{D})<\mu$. By transfinite recursion we then define closed sets $H_{\alpha}$ and points $x_{\alpha}$ for all $\alpha<\varrho$ such that the $H_{\alpha}$ 's are decreasing and the following three conditions are satisfied:

(1) $\psi\left(H_{\alpha}, X\right)<\mu$,

(2) $p \in H_{\alpha}$ and $x_{\alpha} \in H_{\alpha} \backslash\{p\}$,

(3) $H_{\alpha} \cap \overline{\left\{x_{\beta}: \beta<\alpha\right\}} \subset\{p\}$.

To see that this can be done, assume that $\alpha<\varrho$ and $H_{\beta}, x_{\beta}$ have been defined for all $\beta<\alpha$ and satisfy conditions (1)-(3). Set $H=\bigcap\left\{H_{\beta}: \beta<\alpha\right\}$. Then $\varrho \leq \operatorname{cf}(\mu)$ and the inductive hypothesis imply $\psi(H, X)<\mu$.

It is also clear from the inductive hypothesis that $D=\left\{x_{\beta}: \beta<\alpha\right\}$ is a free sequence in the subspace $X \backslash\{p\}$ and hence is discrete. So, as (i) fails, we have either $p \notin \bar{D}$ or $\psi(p, \bar{D})<\mu$. By the regularity of $X$, for each open neighbourhood $U$ of $p$ there is a closed $G_{\delta}$-set $V$ such that $p \in V \subset U$, consequently, in either case, we can find a closed set $K$ with $p \in K, \psi(K, X)<\mu$, and $K \cap \bar{D} \subset\{p\}$.

Now, we define $H_{\alpha}=H \cap K$. Then $\psi\left(H_{\alpha}, X\right)<\mu \leq \psi(p, X)$ implies $\{p\} \varsubsetneqq H_{\alpha}$, hence we can pick $x_{\alpha} \in H_{\alpha} \backslash\{p\}$ and complete the recursion step.

Similarly to the above, the sequence $\left\langle x_{\alpha}: \alpha<\varrho\right\rangle$ is free in $X \backslash\{p\}$, hence $D=\left\{x_{\alpha}: \alpha<\varrho\right\}$ is discrete. We claim that $D \rightarrow p$, hence (ii) holds. Indeed, for any open neighbourhood $U$ of $p$ the set $A=\left\{\alpha: x_{\alpha} \notin U\right\}$ has cardinality less than $\widehat{F}(X) \leq \varrho$ because $\left\langle x_{\alpha}: \alpha \in A\right\rangle$ is a free sequence in $X$ (not just in $X \backslash\{p\}$ ).

As an immediate consequence of Lemma 2.2 we obtain the following strengthening of the deep result $6.14 \mathrm{~b}$ ) from [12].

Corollary 2.3. If $\widehat{F}(X) \leq \lambda=\operatorname{cf}(\lambda) \leq \chi(p, X)$ for a point $p$ in a compactum $X$ then there is a discrete subset $D \subset X$ with $|D| \leq \lambda$ such that $\chi(p, D \cup\{p\}) \geq \lambda$.

As is pointed out in [10, Corollary 3.4], this readily implies that the character $\chi$ reflects all infinite cardinals $\lambda$ for the class of compacta. This means that if $\chi(X) \geq \lambda$ for a compactum $X$ then $\chi(Y) \geq \lambda$ for some $Y \subset X$ with $|Y| \leq \lambda$. If $\lambda$ is regular then 2.3 implies the following strengthening of this reflection result. 
COROllary 2.4. If $\chi(p, X) \geq \lambda=\operatorname{cf}(\lambda)$ for a point $p$ in a compactum $X$ then there is a point $q \in X$ and a discrete $D \subset X$ with $|D| \leq \lambda$ such that $\chi(q, D \cup\{q\}) \geq \lambda$.

Proof. Indeed, if $\widehat{F}(X)>\lambda$ then the main result of [17] yields a free sequence of length $\lambda$ converging to some point $q \in X$, and if $\widehat{F}(X) \leq \lambda$ then Corollary 2.3 applies.

We think the particular case $\lambda=\omega_{1}$ of Corollary 2.4 is worth formulating explicitly: If a compactum is not first countable then it has a non-first countable subspace with a single non-isolated point and of size at most $\omega_{1}$.

We now turn to the main result of this section. For this we need to recall that for any space $X$ the cardinal function $\widehat{t}(X)$ is defined as the smallest cardinal $\kappa$ such that for any set $A \subset X$ we have

$$
\bar{A}=\bigcup\left\{\bar{B}: B \in[A]^{<\kappa}\right\} .
$$

Thus, a non-discrete space $X$ is countably tight iff $\widehat{t}(X)=\omega_{1}$. It is wellknown that if $X$ is a compactum then $\widehat{t}(X) \leq \widehat{F}(X) \leq \widehat{t}(X)^{+}$and if $\widehat{t}(X)$ is regular then $\widehat{t}(X)=\widehat{F}(X)$ (see e.g. 3.12 of [12]).

We also need the following simple result concerning the cardinal function $\widehat{t}(X)$ on compacta.

Lemma 2.5. Let $X$ be a compactum and $\lambda$ be a cardinal with

$$
\lambda=\lambda^{<\widehat{t}(X)}=\sum\left\{\lambda^{\kappa}: \kappa<\widehat{t}(X)\right\} .
$$

Then for every set $Y \subset X$ with $|Y| \leq \lambda$ we have $w(\bar{Y}) \leq \lambda$.

Proof. By the definition of $\widehat{t}(X)$ we have $\bar{Y}=\bigcup\left\{\bar{Z}: Z \in[Y]^{<\widehat{t}(X)}\right\}$. But $\left|[Y]^{<\widehat{t}(X)}\right| \leq \lambda^{<\widehat{t}(X)}=\lambda$ and for each $Z \in[Y]^{<\widehat{t}(X)}$ we have $w(\bar{Z}) \leq 2^{|Z|} \leq$ $2^{<\widehat{t}(X)} \leq \lambda$, hence $n w(\bar{Y}) \leq \lambda$, where $n w$ denotes net weight. As weight equals net weight for compacta by a classical result of Arhangel'skii, this completes the proof.

Now we are ready to formulate and prove our promised result.

TheOREM 2.6. Suppose that $X$ is a compactum, $\lambda$ is a cardinal satisfying $\lambda=\lambda^{<\widehat{t}(X)}$, and $p \in X$ has character $\chi(p, X)>\lambda$. Then $\lambda \in \chi S(p, X)$.

Proof. Note first that $\lambda=\lambda^{<\widehat{t}(X)}$ and $\lambda^{\operatorname{cf}(\lambda)}>\lambda$ clearly imply $\widehat{t}(X) \leq$ $\operatorname{cf}(\lambda) \leq \lambda$. From this we may deduce $\widehat{F}(X) \leq \lambda$. Indeed, to see this we may use $\widehat{F}(X) \leq \widehat{t}(X)^{+}$if $\widehat{t}(X)<\lambda$. If, on the other hand, $\widehat{t}(X)=\lambda$ then $\lambda=\lambda^{<\lambda}$ implies that $\lambda$ is regular, hence $\widehat{t}(X)=\widehat{F}(X)=\lambda$.

CASE 1: $\lambda$ is regular. We may now apply our main Lemma 2.2 for $X$ and $p$ with $\varrho=\lambda$ and $\mu=\lambda^{+}$. Alternative (i) of Lemma 2.2 cannot hold 
because, by Lemma 2.5 , we have

$$
\psi(p, \bar{D})=\chi(p, \bar{D}) \leq w(\bar{D}) \leq \lambda<\mu
$$

whenever $|D|<\lambda$. Consequently, there is a (discrete) set $D$ of size $\lambda$ such that $D \rightarrow p$. Since $\lambda$ is regular, this implies $\chi(p, \bar{D}) \geq \lambda$. On the other hand, by Lemma 2.5 again, we have $\chi(p, \bar{D}) \leq w(\bar{D}) \leq \lambda$, hence $\chi(p, \bar{D})=\lambda$.

CASE $2: \lambda$ is singular. Let us start by fixing a strictly increasing sequence of regular cardinals $\left\{\kappa_{\alpha}: \alpha<\operatorname{cf}(\lambda)\right\}$ converging to $\lambda$ with $\widehat{t}(X) \leq \operatorname{cf}(\lambda)<\kappa_{0}$ and hence $\widehat{F}(X) \leq \kappa_{0}$.

Then, for each $\alpha<\operatorname{cf}(\lambda)$, we may apply Lemma 2.2 to $X$ and $p$ with $\varrho=\kappa_{\alpha}$ and $\mu=\lambda^{+}$. Again, alternative (i) of Lemma 2.2 cannot hold because, by Lemma 2.5, we have $w(\bar{D}) \leq \lambda$ for any $D \subset X$ with $|D|<\kappa_{\alpha}$. Consequently, there is a (discrete) set $D_{\alpha}$ of size $\kappa_{\alpha}$ such that $D_{\alpha} \rightarrow p$.

Now set $Y=\bigcup\left\{D_{\alpha}: \alpha<\operatorname{cf}(\lambda)\right\}$. Then $D_{\alpha} \rightarrow p$ implies $\chi(p, \bar{Y}) \geq \kappa_{\alpha}$ for all $\alpha<\operatorname{cf}(\lambda)$, hence $\chi(p, \bar{Y}) \geq \lambda$. On the other hand, we have $|Y|=\lambda$, so Lemma 2.5 implies $\chi(p, \bar{Y}) \leq w(\bar{Y}) \leq \lambda$, hence $\chi(p, \bar{Y})=\lambda$.

Below we formulate separately the important special case $\widehat{t}(X)=\omega_{1}$ of our theorem.

COROLlary 2.7. If $X$ is a countably tight compactum and $p \in X$ has character $\chi(p, X)>\lambda=\lambda^{\omega}$ then $\lambda \in \chi S(p, X)$.

COROLlary 2.8. If $X$ is any compactum of character $\chi(X)>2^{\omega}$ then either $\omega_{1} \in \chi S(X)$ or $\left\{2^{\omega},\left(2^{\omega}\right)^{+}\right\} \subset \chi S(X)$.

Proof. Indeed, if $X$ is not countably tight then $\omega_{1} \in \chi S(X)$ by Proposition 2.1. Otherwise Corollary 2.7 implies both $2^{\omega} \in \chi S(X)$ and $\left(2^{\omega}\right)^{+} \in$ $\chi S(X)$.

Corollary 2.9. If the compactum $X$ is not first countable then

$$
\chi S(X) \cap\left[\omega_{1}, 2^{\omega}\right] \neq \emptyset .
$$

Proof. If $\chi(X) \leq 2^{\omega}$ then we have a point $p \in X$ with $\omega_{1} \leq \chi(p, X) \leq 2^{\omega}$. Otherwise, Corollary 2.8 implies that $\chi S(X)$ includes at least one of the end points of the closed interval $\left[\omega_{1}, 2^{\omega}\right]$.

It is obvious from our last result that if $2^{\omega}<\aleph_{\omega}$ then the character (and hence the convergence) spectrum of any non-first countable compactum contains a successor and so an uncountable regular cardinal.

COROLlary 2.10. If $\kappa$ is a strong limit cardinal then for every compactum $X$ with $|X| \geq \kappa$ we have

$$
\sup (\kappa \cap \chi S(X))=\kappa .
$$

Proof. First, if $\kappa \leq \widehat{F}(X)$ then, by Proposition 2.1, $\varrho \in \chi S(X)$ for every uncountable regular $\varrho<\kappa$. So assume now that $\tau=\widehat{t}(X) \leq \widehat{F}(X)<\kappa$. 
It follows from Arhangel'skii's theorem and the strong limit property of $\kappa$ that for every cardinal $\lambda<\kappa$ there is a point $p \in X$ such that $\chi(p, X)>\lambda^{\tau}$. But then Theorem 2.6 implies $\lambda^{\tau} \in \kappa \cap \chi S(p, X)$.

Before giving some further results, it is convenient to introduce the concept of the discrete convergence spectrum. This is an obvious variation of the convergence spectrum: The discrete convergence spectrum of the point $p$ in the space $X$ is defined by

$$
d c S(p, X)=\{|D|: D \subset X \text { is discrete and } D \rightarrow p\} .
$$

We also define the global version of this:

$$
d c S(X)=\bigcup\{d c S(x, X): x \in X\},
$$

and we call it the discrete convergence spectrum of $X$.

Unlike the character and hence the convergence spectrum, the discrete convergence spectrum of a compactum may be empty, at least consistently. This is shown for instance by Fedorchuk's compact S-space constructed from $\diamond$ in 7 ].

On the other hand, if $\left\langle x_{\alpha}: \alpha<\kappa\right\rangle$ is a free sequence converging to a point $p$ in a compactum $X$ then both $\kappa$ and $\operatorname{cf}(\kappa)$ belong to $\operatorname{dc} S(X)$. In particular, every compactum that is not countably tight has $\omega_{1}$ in its discrete convergence spectrum. By Balogh's celebrated result in [4], under PFA every countably tight compactum is sequential, and hence has a convergent $\omega$-sequence. Consequently, under PFA, $d c S(X) \cap\left\{\omega, \omega_{1}\right\} \neq \emptyset$ for every compactum $X$.

It is worth mentioning here that in the proof of Theorem 2.6, in addition to $\lambda \in \chi S(p, X) \subset c S(p, X)$, we also obtained $\lambda \in d c S(p, X)$, provided that $\lambda$ is regular.

TheOREM 2.11. Suppose that $X$ is a compactum and $\lambda$ is an uncountable regular cardinal such that $\widehat{F}(X) \leq \lambda$. If a point $p \in X$ has character

$$
\chi(p, X) \geq \sum\left\{\left(2^{\kappa}\right)^{+}: \kappa<\lambda\right\}
$$

then $\lambda \in d c S(p, X)$.

Proof. Set $\mu=\sum\left\{\left(2^{\kappa}\right)^{+}: \kappa<\lambda\right\}$. Then $\operatorname{cf}(\mu) \geq \lambda$ because either $\lambda$ is a limit cardinal and the exponentiation function $2^{\kappa}$ increases for cofinally many $\kappa<\lambda$, in which case $\operatorname{cf}(\mu)=\lambda$, or there is a $\kappa^{*}<\lambda$ with $2^{\kappa}=2^{\kappa^{*}}$ whenever $\kappa^{*} \leq \kappa<\lambda$, and in this case $\mu=\left(2^{\kappa^{*}}\right)^{+}$satisfies $\operatorname{cf}(\mu)>\kappa$ for all $\kappa<\lambda$.

This means that we may apply Lemma 2.2 for $X$ and $p$ with $\varrho=\lambda$ and $\mu$. But for every $D \subset X$ with $|D|<\lambda$ we have $w(\bar{D}) \leq 2^{|D|}<\mu$, which implies that alternative (i) of Lemma 2.2 cannot hold, hence we have (ii), which is exactly what we want. 
COROLlarY 2.12. If $X$ is any compactum and $\lambda$ is an uncountable regular cardinal such that

$$
\chi(p, X) \geq \sum\left\{\left(2^{\kappa}\right)^{+}: \kappa<\lambda\right\}
$$

for some $p \in X$, then $\lambda \in d c S(X)$.

Proof. If $\widehat{F}(X)>\lambda$, i.e. there is a free sequence of length $\lambda$ in $X$, then by the main result of [17] there is also one that converges and we are done. If, on the other hand, $\widehat{F}(X) \leq \lambda$ then we may apply Theorem 2.11 to conclude $\lambda \in d c S(p, X)$.

It is again worth while to formulate explicitly the particular case $\lambda=\kappa^{+}$ of this result:

COROLlary 2.13. If a compactum $X$ has character $\chi(X)>2^{\kappa}$ then $\kappa^{+} \in d c S(X)$.

3. Omission by spectra. Undoubtedly, the most widely known instance of omission of a cardinal by a convergence or character spectrum of a compactum is the fact that $\omega \notin c S(\beta \mathbb{N})$. In general, we say that a set of cardinals $S$ omits a cardinal $\kappa$ if $\kappa \notin S$ but there is a $\lambda \in S$ with $\lambda>\kappa$.

Since $\chi(\beta \mathbb{N})=2^{\omega}$ it follows that $\chi S(\beta \mathbb{N})=c S(\beta \mathbb{N})=\left\{\omega_{1}\right\}$ under $\mathrm{CH}$. Actually, $\mathrm{CH}$ can be weakened to get such a compactum: Fedorchuk constructed in [8] a compactum $X$ satisfying $\chi S(X)=c S(X)=\left\{\omega_{1}\right\}$ from the assumption that the splitting number s equals $\omega_{1}$.

On the other hand, if the pseudo-intersection number $\mathbf{p}$ satisfies $\mathbf{p}>\omega_{1}$ then there is no compactum $X$ whose character spectrum is $\left\{\omega_{1}\right\}$. Indeed, let $x$ be a non-isolated point of a countable set $S \subset X$. Now, if $\chi(x, S)<\mathbf{p}$ then, as is well-known, some $\omega$-sequence from $S \backslash\{x\}$ converges to $x$, hence $\omega \in \chi S(x, X)$. Otherwise, $\chi(x, S) \geq \mathbf{p}>\omega_{1}$ witnesses that $\chi S(X) \neq\left\{\omega_{1}\right\}$.

We note that Hušek's problem mentioned in the introduction is equivalent to asking if there is a compactum $X$ whose convergence spectrum $c S(X)$ omits both $\omega$ and $\omega_{1}$.

Now, consider the assumption that the character spectrum $\chi S(X)$ of a compactum $X$ omits both $\omega$ and $\omega_{1}$. Then, by Proposition 2.1, $X$ is countably tight. It follows that $X^{\prime}$, the derived set of $X$, has no isolated point because such a point would have a neighbourhood homeomorphic to the onepoint compactification of an infinite discrete space and hence would include a converging $\omega$-sequence. Consequently, $X^{\prime}$ is a countably tight compactum in which every point has character at least $\omega_{2}$, i.e. $X^{\prime}$ is a counterexample to the conjecture from [13] that was mentioned in the introduction.

Next, we shall study the question if one can find compacta whose character spectra omit uncountable cardinals. By Corollary 2.9, CH implies that $\omega_{1}$ cannot be omitted by $\chi S(X)$ for any compactum $X$. On the other hand, 
Hušek showed in [11] that the converse of this is also true by constructing, in ZFC, a compactum $X$ such that $\chi S(X)=\left\{\omega, 2^{\omega}\right\}$.

Below we shall give a general method for constructing (consistent) examples of compacta whose character spectra omit various uncountable cardinals. Using this method we shall show, among other things, that Hušek's example can be easily obtained from an earlier construction of van Douwen.

First, however, we recall the perhaps most basic spectrum of a topological space $X$ that was introduced and studied in [14]: the cardinality spectrum $S(X)$ of a space $X$ is the set of cardinalities of all infinite closed subspaces of $X$. Note that for a compactum $X$ this is the same as the set of sizes of all subcompacta of $X$.

Lemma 3.1. Let $Y$ be a locally compact $T_{2}$ space which is also locally $\mu$, that is, every point of $Y$ has a neighbourhood of cardinality at most $\mu$, and let $X=Y \cup\{p\}$ be the one-point compactification of $Y$. If $\mu<\kappa<|Y|$ and $\kappa \notin S(Y)$ then $\kappa \notin \chi S(X)$.

Proof. First, observe that every compact subset of $Y$ also has cardinality at most $\mu$, hence $|X \backslash U| \leq \mu$ for every neighbourhood $U$ of $p$ in $X$. This clearly implies that $\psi(p, X)=\chi(p, X)=|F|$ whenever $p$ is a non-isolated point of a closed subset $F$ of $X$ with $|F|>\mu$. But we have $\chi(p, X) \leq|F| \leq \mu$ otherwise, hence $\kappa \notin \chi S(p, X)$. As trivially $\chi(x, X)=\chi(x, Y) \leq \mu$ for all points $x \in Y$, we may indeed conclude that $\kappa \notin \chi S(X)$.

Note that, as $|X|=|Y| \in \chi S(X)$, this actually means that $\kappa$ is omitted by $\chi S(X)$.

In the mid 70's Eric van Douwen invented a technique for constructing a locally compact and locally countable refinement $\Lambda$ of the reals having, among many others, the property $S(\Lambda)=\left\{\omega, 2^{\omega}\right\}$. This construction was widely circulated in handwritten form but, as far as we know, got never published by him. However, an early announcement of the construction was published in [5]. Now, it is immediate from Lemma 3.1 that the one-point compactification of $\Lambda$ has character spectrum $\left\{\omega, 2^{\omega}\right\}$.

COROLlaRY 3.2 (Hušek, [11]). There is a ZFC example of a compactum $X$ such that $\chi S(X)=\left\{\omega, 2^{\omega}\right\}$.

In view of Proposition 2.1, any compactum $X$ whose character spectrum omits $\omega_{1}$ must be countably tight, consequently, by Corollary 2.7, if $\chi S(X)=$ $\{\omega, \kappa\}$ then we must have $\kappa \leq 2^{\omega}$. It is natural to ask if the converse is also true: Does $\omega_{1} \leq \kappa \leq 2^{\omega}$ imply the existence of a compactum whose character spectrum is $\{\omega, \kappa\}$ ? We are now going to study this question and give important partial answers to it.

Definition 3.3. For any cardinal $\kappa \leq 2^{\omega}$ we let $\Phi(\kappa)$ denote the following statement: There are a set of reals $T \in[\mathbb{R}]^{\kappa}$ and a family $\mathcal{A} \subset[T]^{\omega}$ with 
$|\mathcal{A}|=\kappa$ such that (i) for every $A \in \mathcal{A}$ we have $|T \cap \bar{A}|=\kappa$ and (ii) for every $B \in[T]^{\omega_{1}}$ there is $A \in \mathcal{A}$ with $A \subset B$.

Note that $\Phi\left(2^{\omega}\right)$ is trivially true, as witnessed by $T=\mathbb{R}$ and $\mathcal{A}=\{A \in$ $\left.[\mathbb{R}]^{\omega}:|\bar{A}|>\omega\right\}$.

LEMma 3.4. $\Phi(\kappa)$ implies that there is a locally countable and locally compact $T_{2}$ space $Y$ with $S(Y)=\{\omega, \kappa\}$ and hence a compactum $X$ with $\chi S(X)=\{\omega, \kappa\}$.

Proof. Assume that $T$ and $\mathcal{A}$ witness $\Phi(\kappa)$. For technical reasons we assume that for some countable dense subset $D$ of $T$ we have $D \in \mathcal{A}$. Fix a $\kappa$-type well-ordering $\prec$ of $T$ such that $D$ is the first $\omega$ elements of $T$ with respect to $\prec$.

Next let $\mathcal{A}=\left\{A_{\alpha}: \alpha<\kappa\right\}$ be an enumeration of $\mathcal{A}$ such that for every $A \in \mathcal{A}$ we have $\left|\left\{\alpha<\kappa: A=A_{\alpha}\right\}\right|=\kappa$.

By a generalization of van Douwen's technique for $\kappa=2^{\omega}$, we are going to construct a locally countable and locally compact topology on $T$ that refines its Euclidean topology $\varepsilon$. This is done by transfinite recursion on $\alpha<\kappa$ as follows.

Assume that $\alpha<\kappa$ and $Y_{\alpha}=\left\{y_{\beta}: \beta<\alpha\right\} \subset T$ and a locally countable, locally compact topology $\tau_{\alpha}$ on $Y_{\alpha}$ refining $\varepsilon \mid Y_{\alpha}$ have been defined in such a way that $Y_{\beta}$ with $\tau_{\beta}$ is an open subspace of $Y_{\alpha}$ for all $\beta<\alpha$.

Now, if $A_{\alpha} \subset Y_{\alpha}$ then we first define $y_{\alpha}$ as the $\prec$-minimal member of the clearly non-empty set $T \cap \overline{A_{\alpha}} \backslash Y_{\alpha}$. By definition, we may then fix a one-to-one sequence $\left\{x_{n}: n<\omega\right\} \subset A_{\alpha} \subset Y_{\alpha}$ that $\varepsilon$-converges to $y_{\alpha}$. By the inductive hypothesis, we can also fix, for each $n<\omega$, a compact open neighbourhood $K_{n}$ of $x_{n}$ with respect to $\tau_{\alpha}$ such that diam $K_{n}<1 / 2^{n}$.

Let us then set $U_{n}=\bigcup_{i>n} K_{i} \cup\left\{y_{\alpha}\right\}$ for all $n<\omega$, and define $\tau_{\alpha+1}$ as the topology on $Y_{\alpha+1}=Y_{\alpha} \cup\left\{y_{\alpha}\right\}$ generated by $\tau_{\alpha} \cup\left\{U_{n}: n<\omega\right\}$. It is standard and easy to show that each $U_{n}$ is compact and open with respect to $\tau_{\alpha+1}$ as well as that $\tau_{\alpha+1} \supset \varepsilon \mid Y_{\alpha+1}$.

If $A_{\alpha}$ is not a subset of $Y_{\alpha}$ then the $\prec$-minimal element of $T \backslash Y_{\alpha}$ is chosen to be $y_{\alpha}$, and it is declared to be an isolated point of $Y_{\alpha+1}$ with respect to $\tau_{\alpha+1}$, that is, $\tau_{\alpha+1}$ is generated by $\tau_{\alpha} \cup\left\{y_{\alpha}\right\}$.

This completes the description of any successor step of the recursion; in the limit steps we simply take direct limits. Finally, we end up with a locally countable and locally compact topology $\tau=\bigcup_{\alpha<\kappa} \tau_{\alpha} \supset \varepsilon$ on $T$. Indeed, the fact that for each $x \in T$ there is an $\alpha<\kappa$ with $x=y_{\alpha}$ follows from $D \in \mathcal{A}$ and $\left|\left\{\alpha<\kappa: D=A_{\alpha}\right\}\right|=\kappa$.

To complete the proof, we have to show that $S(Y)=\{\omega, \kappa\}$ for the space $Y=\langle T, \tau\rangle$. This follows because we have added $\kappa$ many $\tau$-limit points to every bounded member of $\mathcal{A}$, and moreover every uncountable subset $B$ of 
$T$ includes a bounded element of $\mathcal{A}$. The latter statement holds because if $\operatorname{cf}(\kappa)=\omega$ then $B$ has an uncountable bounded subset.

Now we consider a condition that implies $\Phi(\kappa)$ for certain cardinals $\kappa<2^{\omega}$.

LEMma 3.5. If the partial order $\left\langle[\kappa]^{\omega_{1}}, \subset\right\rangle$ has a dense set of size $\kappa$ (we shall denote this by $\left.\pi\left([\kappa]^{\omega_{1}}\right)=\kappa\right)$ for some $\kappa<2^{\omega}$ with $\operatorname{cf}(\kappa) \neq \omega_{1}$ then $\Phi(\kappa)$ holds true.

Proof. Let $\mathcal{B} \subset[\kappa]^{\omega_{1}}$ with $|\mathcal{B}|=\kappa$ be dense in $\left\langle[\kappa]^{\omega_{1}}, \subset\right\rangle$; since $\operatorname{cf}(\kappa) \neq \omega_{1}$ we may assume that every $B \in \mathcal{B}$ is bounded in $\kappa$. It will suffice to construct a set $T=\left\{x_{\alpha}: \alpha<\kappa\right\} \subset \mathbb{R}$ of distinct points such that for every $B \in \mathcal{B}$ the subset $\widetilde{B}=\left\{x_{\xi}: \xi \in B\right\} \subset T$ has $\kappa$ limit points in $T$. Indeed, if for each $B \in \mathcal{B}$ we pick a countable dense subset $A_{B}$ of $\widetilde{B}$ then $T$ together with $\mathcal{A}=\left\{A_{B}: B \in \mathcal{B}\right\}$ clearly witnesses $\Phi(\kappa)$.

Now, to construct $T$ as above fix an enumeration $\left\{B_{\alpha}: \alpha<\kappa\right\}$ of $\mathcal{B}$ such that for every $B \in \mathcal{B}$ we have $\left|\left\{\alpha<\kappa: B=B_{\alpha}\right\}\right|=\kappa$. The points $x_{\alpha} \in \mathbb{R}$ are picked recursively as follows.

Assume that $\alpha<\kappa$ and $T_{\alpha}=\left\{x_{\beta}: \beta<\alpha\right\}$ have been picked already. If $B_{\alpha} \subseteq \alpha$ then we define $x_{\alpha}$ as any limit point of $\widetilde{B_{\alpha}}$ outside of $T_{\alpha}$. Otherwise, if $\widetilde{B_{\alpha}}$ is not yet defined, we let $x_{\alpha}$ be any element of $\mathbb{R} \backslash T_{\alpha}$. Since every $B \in \mathcal{B}$ is bounded, this will clearly work.

Arnie Miller showed in [21] that if one adds $\aleph_{\omega}$ Cohen reals to a ground model satisfying GCH then $\pi\left(\left[\aleph_{\omega}\right]^{\omega_{1}}\right)=\aleph_{\omega}$ in the resulting generic extension. In fact, the same argument given in [21] yields the following more general result whose proof we therefore leave to the reader.

Proposition 3.6. Assume that $\lambda$ is a singular cardinal of countable cofinality that is $\omega_{1}$-inaccessible, i.e. $\mu^{\omega_{1}}<\lambda$ whenever $\mu<\lambda$. Then for every CCC partial order $\mathbb{P}$ with $|\mathbb{P}|=\lambda$ the equality $\pi\left([\lambda]^{\omega_{1}}\right)=\lambda$ holds in the generic extension $V^{\mathbb{P}}$.

COROllary 3.7. Assume that GCH holds and $\lambda$ is a singular cardinal of countable cofinality in our ground model $V$. If we add $\lambda$ Cohen reals to $V$ then in the generic extension $\Phi(\lambda)$ holds, hence there is a compactum $X$ such that $\chi S(X)=\{\omega, \lambda\}$.

Note that Corollary 3.7 yields the consistency of the existence of ABcompacta that are not first countable. In fact, let $X=Y \cup\{p\}$ be the onepoint compactification of a locally countable space $Y$. Then every uncountable subset of $Y$ converges to $p$ in $X$, hence the convergence spectrum $c S(X)$ equals $[\omega, \lambda]$, showing that the AB-compactum $X$ is not a K-compactum. Thus Corollary 3.7 answers two questions that were raised in [9]. 
It is probably worth mentioning that the spaces constructed in Corollary 3.7 also yield examples of compacta whose weight spectrum is $\{\omega, \lambda\}$. These examples are very different from the ones constructed in [15] that were first countable.

Motivated by Corollary 3.7, L. Soukup has recently proved the following: It is consistent that the continuum is arbitrarily large and $\Phi(\kappa)$ holds for all $\kappa \leq 2^{\omega}$. This non-trivial result is still unpublished but, luckily, we may avoid using it in proving the following corollary of it: It is consistent that $2^{\omega}$ is arbitrarily large and for every $\kappa \leq 2^{\omega}$ there is a compactum $X$ whose character spectrum $\chi S(X)$ equals $\{\omega, \kappa\}$.

Before doing that, however, we point out that under Martin's axiom, $\Phi(\kappa)$ fails whenever $\omega_{1}<\kappa<2^{\omega}$. Indeed, as is well-known, every subset of any $T \in[\mathbb{R}]^{\kappa}$ is then a relative $F_{\sigma}$ and hence $T$ has a relatively closed subset of size $\omega_{1}$.

The following simple but crucial observation is due to Z. Szentmiklóssy.

LEMMA 3.8. Let $X$ be an arbitrary topological space in the ground model $V$ and $G$ be $\mathbb{C}_{\lambda}=\operatorname{Fn}(\lambda, 2)$ generic over $V$. Then $X$ has the following property in the extension $V[G]$ : For every set $A \in[X]^{\omega_{1}}$ there is an uncountable ground model set $B \in V \cap[X]^{\omega_{1}}$ such that $B^{\prime} \subset \bar{A}$, i.e. every limit point of $B$ is in the closure of $A$.

Proof. Let $\dot{A}$ be a $\mathbb{C}_{\lambda}$ name for $A$ and assume that $p \Vdash \dot{A} \in[X]^{\omega_{1}}$. It is standard to find then a set $B \in V \cap[X]^{\omega_{1}}$ and for each point $x \in B$ a condition $p_{x} \leq p$ forcing $x \in \dot{A}$ such that $\left\{p_{x}: x \in B\right\}$ forms a $\Delta$-system with root $q \leq p$. We claim that $q$ forces $B^{\prime} \subset \overline{\dot{A}}$.

To see this, it suffices to show that for every condition $s \leq q$, every point $y \in B^{\prime}$, and every neighbourhood $U$ of $y$ there is an extension $r$ of $s$ that forces $U \cap \dot{A} \neq \emptyset$.

But as $y$ is a limit point of $B$, the set $U \cap B$ is infinite, consequently there is an $x \in U \cap B$ for which $p_{x}$ and $s$ are compatible. Then $r=s \cup p_{x}$ is clearly as required.

Now, we are ready to formulate and prove the promised result.

TheOREM 3.9. Assume that our ground model $V$ satisfies $G C H$ and $\lambda$ is an infinite cardinal in $V$. Then, in the generic extension $V^{\mathbb{C}_{\lambda}}$, for every $\kappa \leq \lambda$ there is a locally countable and locally compact $T_{2}$ space $Y$ with $S(Y)=\{\omega, \kappa\}$ and hence there is a compactum $X$ with character spectrum $\chi S(X)=\{\omega, \kappa\}$.

Proof. First observe that for every $\kappa>\omega$ the principle $\Phi(\kappa)$ holds in $V^{\mathbb{C}_{\kappa}}$. Indeed, if $\operatorname{cf}(\kappa)>\omega$ then this is because $V^{\mathbb{C}_{\kappa}} \vDash \kappa=2^{\omega}$, and if $\operatorname{cf}(\kappa)=\omega$ then this follows from Corollary 3.7 . 
But, for any $\kappa<\lambda$, forcing with $\mathbb{C}_{\lambda}$ is the same as first forcing with $\mathbb{C}_{\kappa}$ and then with $\mathbb{C}_{\lambda \backslash \kappa}$. By Lemma 3.4 the first forcing produces a locally countable and locally compact $T_{2}$ space $Y$ with $S(Y)=\{\omega, \kappa\}$, and all these properties of $Y$ will be preserved by the second forcing. In fact, the locally countable and locally compact $T_{2}$ properties together are preserved in any extension. Moreover, $S(Y)=\{\omega, \kappa\}$ is preserved because, by Lemma 3.8. the closure of every uncountable subset of $Y$ is of size $\kappa$. Now, by Lemma 3.1. the one-point compactification $X$ of $Y$ satisfies $\chi S(X)=\{\omega, \kappa\}$.

COROLlary 3.10. It is consistent with $2^{\omega}$ as big as you wish that for every countable set $C$ of cardinals with $\omega \in C \subset\left[\omega, 2^{\omega}\right]$ there is a compactum $X$ such that $\chi S(X)=C$.

Proof. Consider the model of Theorem 3.9 for any regular cardinal $\lambda$ and fix for each $\kappa \leq \lambda$ a compactum $X_{\kappa}$ such that $\chi S\left(X_{\kappa}\right)=\{\omega, \kappa\}$. If $C$ is finite then we may simply take $X$ to be the topological sum of the spaces $\left\{X_{\kappa}: \kappa \in C\right\}$. If $C$ is infinite then the one-point compactification of the topological sum of $\left\{X_{\kappa}: \kappa \in C\right\}$ will work.

We next examine the question what, if any, restrictions follow for a compactum whose character or convergence spectrum omits certain cardinals. For instance, I. Gorelic, in a personal communication, raised the question if there is an upper bound for the size of a K-compactum. One of the main results of [9] was the following theorem that answered Gorelic's question: The cardinality of any AB-compactum $X$ is at most $2^{<2^{\omega}}$. The proof relies on showing that $|X| \leq\left(\sup \left\{|\bar{S}|: S \in[X]^{\omega}\right\}\right)^{\omega}$. Our next result shows that this inequality actually holds for a significantly wider class of compacta, namely those whose character spectra contain only limit cardinals.

THEOREM 3.11. If $X$ is a compactum such that every $\lambda \in \chi S(X)$ is a limit cardinal then $|X| \leq\left(\sup \left\{|\bar{S}|: S \in[X]^{\omega}\right\}\right)^{\omega}$.

Proof. Observe first of all that as $\omega_{1} \notin \chi S(X)$, by Proposition 2.1, $X$ is countably tight. Set $\mu=\left(\sup \left\{|\bar{S}|: S \in[X]^{\omega}\right\}\right)^{\omega}$. Then we have both $\mu=\mu^{\omega}$ and $\mu^{+}=\left(\mu^{+}\right)^{\omega}$. It follows from the second equality and Corollary 2.7 that $\chi(x, X) \leq \mu$ for every point $x \in X$. So fix for each $x \in X$ a neighbourhood base $\mathcal{B}_{x}$ with $\left|\mathcal{B}_{x}\right| \leq \mu$.

Let $\vartheta$ be a large enough regular cardinal such that the space $X$ (and everything else that is relevant, e.g. the map $x \mapsto \mathcal{B}_{x}$ ) belongs to $H(\vartheta)$, the structure of all sets hereditarily of size $<\vartheta$.

Using $\mu=\mu^{\omega}$ we can find a countably closed elementary submodel $M \prec$ $H(\vartheta)$ of cardinality $\mu$ that again contains everything relevant and satisfies $\mu \subset M$. Note that $\mathcal{B}_{x} \subset M$ for each $x \in X \cap M$ because $\left|\mathcal{B}_{x}\right| \leq \mu$.

We claim that then $X \subset M$, and that will complete our proof. To see the claim, first note that $X \cap M$ is a closed subset of $X$. Indeed, for every 
countable set $S \subset X \cap M$ we have $S \in M$ because $M$ is countably closed, hence $\bar{S} \in M$ as well. But $|\bar{S}| \leq \mu$ by definition, and consequently $\bar{S} \subset$ $X \cap M$. Since $X$ is countably tight, this indeed implies that $X \cap M$ is closed.

Now, assume that, say, $q \in X \backslash M$. Then every point $x \in X \cap M$ has a neighbourhood $U_{x} \in \mathcal{B}_{x}$ such that $q \notin U_{x}$. But $X \cap M$ is compact, being closed in $X$, hence there are finitely many points $x_{1}, \ldots, x_{n} \in X \cap M$ for which $U_{x_{1}} \cup \cdots \cup U_{x_{n}}$ covers $X \cap M$. However, as each $U_{x_{i}}$ is in $M$, this would imply $U_{x_{1}} \cup \cdots \cup U_{x_{n}}=X$, which clearly is a contradiction.

Since every separable compactum has cardinality at most $2^{2^{\omega}}$, it is immediate from our last result that if every $\lambda \in \chi S(X)$ is a limit cardinal for a compactum $X$ then $|X| \leq 2^{2^{\omega}}$. However, this already follows from Corollary 2.8 . just assuming that neither $\omega_{1}$ nor $\left(2^{\omega}\right)^{+}$belongs to $\chi S(X)$. Indeed, then we may conclude $\chi(X) \leq 2^{\omega}$ and apply Arhangel'skii's theorem.

Similarly, Corollary 2.8 implies that if $\left\{\omega_{1}, 2^{\omega}\right\} \cap \chi S(X)=\emptyset$ for a compactum $X$ then $\chi(X) \leq 2^{\omega}$, and hence $|X| \leq 2^{2^{\omega}}$. So the following natural question arises: If the character spectrum of a compactum $X$ omits all uncountable cardinals strictly below $2^{\omega}$, does that put a bound on the size (or character) of $X$ ? Perhaps surprisingly, the answer to this question is, consistently, negative, as can be seen from our next result.

THEOREM 3.12. Let $\kappa$ be any uncountable regular cardinal and $\mathbb{P}_{\kappa}$ be the standard CCC partial order that iteratively adds $\kappa$ dominating (or Hechler) reals to the ground model $V$. Then, in $V^{\mathbb{P}_{\kappa}}$, there are arbitrarily large compacta whose character spectra omit all members of the open interval $(\omega, \kappa)$.

Proof. It was shown in [1] for the particular case $\kappa=\omega_{1}$ that, in $V^{\mathbb{P}_{\kappa}}$, there are arbitrarily large Jakovlev spaces. However, it is straightforward to check that essentially the same argument yields the same conclusion for any uncountable regular cardinal $\kappa$ (more details of this will appear in [18]).

Now, all the reader has to know about Jakovlev spaces is that they are locally countable and locally compact $T_{2}$ spaces, every closed subspace of a Jakovlev spaces is Jakovlev, and any uncountable Jakovlev space has size at least the bounding number $\mathbf{b}$ (see [1]). Consequently, the cardinality spectrum of any uncountable Jakovlev space omits all members of the interval $(\omega, \mathbf{b})$, and then by Lemma 3.1 so does the character spectrum of its one-point compactification.

Finally, it is obvious that for a regular $\kappa$ we have $\mathbf{b}=\kappa$ in the generic extension $V^{\mathbb{P}_{\kappa}}$.

While Theorem 3.12 shows that e.g. $\omega_{1} \notin \chi S(X)$ does not put any bound on the size of a compactum $X$, it is immediate from Corollary 2.13 that even $\omega_{1} \notin d c S(X)$ implies $\chi(X) \leq 2^{\omega}$ and hence $|X| \leq 2^{2^{\omega}}$. 
We have so far produced numerous (consistent) examples of compacta whose character spectra omit lots of uncountable cardinals. All these examples were one-point compactifications of locally countable, locally compact spaces and therefore, as we already pointed out, their convergence spectra did not omit any cardinal. Theorem 3.14 below provides easy examples of compacta whose convergence spectra, under suitable cardinal arithmetic assumptions, omit lots of cardinals. First, however, we present a lemma that may be of independent interest.

Lemma 3.13. Assume that $X$ is a compactum, $\lambda$ is a singular cardinal, and $S \in[X]^{\lambda}$ converges to $p \in X$. If, in addition, $\operatorname{cf}(\lambda) \notin c S(p, X)$ then there is a $\mu<\lambda$ such that for every neighbourhood $U$ of $p$ we have $|S \backslash U|<\mu$. Consequently, $[\mu, \lambda] \subset c S(p, X)$.

Proof. For every cardinal $\kappa<\lambda$ set

$$
G_{\kappa}=\bigcup\{U: U \text { open, }|U \cap S|<\kappa\} .
$$

Since $S \rightarrow p$ and $X$ is Hausdorff, we clearly have

$$
\bigcup\left\{G_{\kappa}: \kappa<\lambda\right\}=X \backslash\{p\} .
$$

In fact, we claim that there is some $\mu<\lambda$ for which $G_{\mu}=X \backslash\{p\}$.

Assume that this fails. Then by a straightforward transfinite recursion we may define cardinals $\left\{\kappa_{\alpha}: \alpha<\operatorname{cf}(\lambda)\right\}$ converging up to $\lambda$ and distinct points $\left\{x_{\alpha}: \alpha<\operatorname{cf}(\lambda)\right\}$ such that $x_{\alpha} \notin G_{\kappa_{\alpha}}$ whenever $\alpha<\operatorname{cf}(\lambda)$.

Now let $V$ be any closed neighbourhood of $p$. Then $\left|\left\{\alpha: x_{\alpha} \notin V\right\}\right|<$ $\operatorname{cf}(\lambda)$ because otherwise we would have $|S \backslash V|=\lambda$. As $X$ is also regular, this would imply $\left\{x_{\alpha}: \alpha<\operatorname{cf}(\lambda)\right\} \rightarrow p$, contradicting $\operatorname{cf}(\lambda) \notin c S(p, X)$.

TheOREM 3.14. Let $X$ be a crowded (i.e. dense-in-itself) compactum such that $\chi S(X)=\{\kappa\}$ where $\kappa \in\left\{\omega, \omega_{1}\right\}$. Then

$$
c S(p, X)=\{\mu<|X|: \operatorname{cf}(\mu)=\kappa\} \quad \text { for every } p \in X .
$$

Proof. We first consider the case $\kappa=\omega$. Clearly then $|G|=\mathbf{c}=2^{\omega}$ for every non-empty open set $G$ in $X$. For any cardinal $\mu$ such that $\omega<\mu<\mathbf{c}$ and $\operatorname{cf}(\mu)=\omega$ pick a sequence of cardinals $\mu_{n}$ that converges up to $\mu$. For any point $p \in X$ we can fix an open neighbourhood base $\left\{U_{n}: n<\omega\right\}$ of $p$ such that $\overline{U_{n+1}} \subsetneq U_{n}$ for all $n<\omega$. Then for every $n<\omega$ we can select a subset $A_{n} \subset U_{n} \backslash \overline{U_{n+1}}$ with $\left|A_{n}\right|=\mu_{n}$. Obviously, then $A=\bigcup_{n<\omega} A_{n} \rightarrow p$ and $|A|=\mu$, hence $\mu \in c S(p, X)$. The fact that no $\lambda$ with $\operatorname{cf}(\lambda)>\omega$ belongs to $c S(p, X)$ is obvious from $\chi(p, X)=\omega$.

Now, assume that $\kappa=\omega_{1}$. It follows from the classical theorems of Cech-Pospišil and Arhange'lskii that $|X|=2^{\omega_{1}}$. In fact, we also have $|H|=$ $2^{\omega_{1}}$ for every non-empty $G_{\delta}$-set $H$. For closed $G_{\delta}$-sets this follows from 
$\chi S(X)=\left\{\omega_{1}\right\}$ by the same argument, and for the general case because every non-empty $G_{\delta}$-set includes one that is closed.

For any point $p \in X$ fix an open neighbourhood base $\left\{U_{\alpha}: \alpha<\omega_{1}\right\}$. It is then straightforward to define a closed $G_{\delta}$-set for each $\alpha<\omega_{1}$ in such a way that $p \in H_{\alpha} \subset U_{\alpha}$ and $H_{\beta} \subsetneq H_{\alpha}$ whenever $\alpha<\beta<\omega_{1}$. Now, if $\omega_{1}<\mu<2^{\omega_{1}}$ with $\operatorname{cf}(\mu)=\omega_{1}$ then we fix a sequence $\left\{\mu_{\alpha}: \alpha<\omega_{1}\right\}$ of cardinals that converges up to $\mu$. Similarly to the previous case, we may then choose a subset $A_{\alpha} \subset H_{\alpha} \backslash H_{\alpha+1}$ with $\left|A_{\alpha}\right|=\mu_{\alpha}$ and conclude that $\bigcup_{\alpha<\omega_{1}} A_{\alpha} \rightarrow p$, hence $\mu \in c S(p, X)$.

That no $\lambda$ with $\operatorname{cf}(\lambda)>\omega_{1}$ belongs to $c S(p, X)$ is again obvious from $\chi(p, X)=\omega_{1}$. Finally, if $\lambda$ satisfies $\operatorname{cf}(\lambda)=\omega$ then $\omega \notin \chi S(X)$ implies $\omega \notin c S(X)$. Now we may apply Lemma 3.13 to conclude that $\lambda \notin c S(X)$ since otherwise we would have a regular cardinal $\varrho \in c S(X)$ with $\omega_{1}<\varrho<\lambda$, which we already know is impossible.

Note that, at least so far, $\chi S(X)=\{\kappa\}$ is known to be consistent only for $\kappa \in\left\{\omega, \omega_{1}\right\}$. And if the conjecture that $\min \chi S(X) \leq \omega_{1}$ is provable in ZFC turns out to be correct then $\chi S(X) \neq\{\kappa\}$ for any other $\kappa$, for any compactum $X$. Also, the assumption that $X$ be crowded is essential if $\kappa=\omega$, as is shown by any countable scattered compactum.

It is immediate from Theorem 3.14 that under $\mathbf{c}>\aleph_{\omega}$ or under $\mathbf{s}=\omega_{1}$ and $2^{\omega_{1}}>\aleph_{\omega_{1}}$ there are compacta whose convergence spectra omit lots of cardinals. However, all these spectra are such that, except their minima, all their members are singular cardinals. It seems to be much harder to find compacta $X$ such that $c S(X) \cap \mathrm{REG}$ omits a regular cardinal.

We know only one such example, namely a non-first countable compactum whose convergence spectrum omits $\omega_{1}$. It is the one-point compactification $X=Y \cup\{p\}$ of the first countable, locally compact, initially $\omega_{1^{-}}$ compact but non-compact space $Y$ of cardinality $\omega_{2}$ that was constructed, with a considerable effort, in [16]. Indeed, if $A \in[Y]^{\omega_{1}}$ then $A$ cannot converge to any $y \in Y$ because $y$ has countable character and $A$ cannot converge to $p$ because it has a complete accumulation point in $Y$, hence $\omega_{1} \notin c S(X)$. In fact, as $\chi(p, X)=\omega_{2}$, we have $\chi S(X)=c S(X)=\left\{\omega, \omega_{2}\right\}$. Just for curiosity, we mention that $Y$ has no discrete subset of size $\omega_{2}$, hence $d c S(p, X)=\emptyset$ and $d c S(X)=\{\omega\}$.

Acknowledgments. The first author was supported by OTKA grants no. 61600 and 68262 .

\section{References}

[1] U. Abraham, I. Gorelic, and I. Juhász, On Jakovlev spaces, Israel J. Math. 152 (2006), 205-219. 
[2] A. V. Arhangel'skii and R. Z. Buzyakova, Convergence in compacta and linear Lindelöfness, Comment. Math. Univ. Carolin. 39 (1998), 159-166.

[3] L. Babai and A. Máté, Inner set mappings on locally compact spaces, in: Topics in Topology (Keszthely, 1972), Colloq. Math. Soc. János Bolyai 8, North-Holland, Amsterdam, 1974, 77-95.

[4] Z. Balogh, On compacta of countable tightness, Proc. Amer. Math. Soc. 105 (1989), 755-764.

[5] E. K. van Douwen, A technique for constructing examples, in: General Topology and Its Relations to Modern Analysis and Algebra, IV (Praha, 1976), J. Novák (ed.), Part B, Soc. Czech. Math. Phys., Praha, 1977, 111-112.

[6] A. Dow, Compact spaces of countable tightness in the Cohen model, in: Set Theory and Its Applications, Lecture Notes in Math. 1401, Springer, 1989, 55-67.

[7] V. V. Fedorchuk, On the cardinality of hereditarily separable compacta, Soviet Math. Dokl. 16 (1975), 651-655.

[8] - A compact space having cardinality continuum with no convergent sequences, Math. Proc. Cambridge Philos. Soc. 81 (1977), 177-181.

[9] I. Gorelic and I. Juhász, AB-compacta, Comment. Math. Univ. Carolin. 49 (2008), 141-146.

[10] R. E. Hodel and J. E. Vaughan, Reflection theorems for cardinal functions, Topology Appl. 100 (2000), 47-66.

[11] M. Hušek, Omitting cardinal functions by topological spaces, in: General Topology and Its Relations to Modern Analysis and Algebra, V (Praha, 1981), J. Novák (ed.), Heldermann, Berlin, 1983, 387-394.

[12] I. Juhász, Cardinal Functions in Topology - Ten Years Later, Math. Centre Tracts 123, Math. Centrum, Amsterdam, 1980.

[13] - On the minimum character of points in compact spaces, in: Topology. Theory and Applications (Pécs, 1989), Colloq. Math. Soc. János Bolyai 55, North-Holland, 1993, 365-371.

[14] —, The cardinality and weight spectrum of a compact space, in: Recent Developments of General Topology and Applications, in Memory of F. Hausdorff (W. Gähler et al., eds.), Akademie-Verlag, Berlin, 1992, 170-175.

[15] -, On the weight spectrum of a compact space, Israel J. Math. 81 (1993), 369-379.

[16] I. Juhász, P. Koszmider, and L. Soukup, A first countable, initially $\omega_{1}$-compact but non-compact space, Topology Appl. 156 (2009), 1863-1879.

[17] I. Juhász and Z. Szentmiklóssy, Convergent free sequences in compact spaces, Proc. Amer. Math. Soc. 116 (1992), 1153-1160.

[18] I. Juhász and W. Weiss, Large Jakovlev spaces, in preparation.

[19] K. Kunen, Locally compact linearly Lindelöf spaces, Comment. Math. Univ. Carolin. 43 (2002), 155-158.

[20] —, Small locally compact linearly Lindelöf spaces, Topology Proc. 29 (2005), 193198.

[21] A. W. Miller, The Baire category theorem and cardinals of countable cofinality, J. Symbolic Logic 47 (1982), 275-288.

István Juhász

Alfréd Rényi Institute of Mathematics

H-1053 Budapest, Hungary

E-mail: juhasz@renyi.hu
William A. R. Weiss Department of Mathematics

University of Toronto Toronto, Ontario, Canada M5S 2E4

E-mail: weiss@math.toronto.edu

Received 22 July 2009;

in revised form 10 October 2009 